OPEN ACCESS

Edited by:

Akio Adachi,

Kansai Medical University, Japan

Reviewed by:

Zsolt Ruzsics,

University of Freiburg Medical

Center, Germany

*Correspondence:

Graciela Andrei

graciela.andrei@kuleuven.be

Specialty section:

This article was submitted to

Antivirals and Vaccines,

a section of the journal

Frontiers in Virology

Received: 10 February 2021 Accepted: 20 April 2021

Published: 24 May 2021

Citation:

Andrei G (2021) Vaccines and Antivirals: Grand Challenges and

Great Opportunities.

Front. Virol. 1:666548

doi: 10.3389/fviro.2021.666548

\section{Vaccines and Antivirals: Grand Challenges and Great Opportunities}

\author{
Graciela Andrei* \\ Laboratory of Virology and Chemotherapy, Rega Institute for Medical Research, KU Leuven, Leuven, Belgium
}

Keywords: vaccines, antivirals, (re)emerging infectious disease, pandemics, epidemics, drug discovery, viral targets

\section{INTRODUCTION}

In the last decades, emerging and reemerging infectious diseases marked the third epidemiological transition (Figure 1A), characterized by: (i) identification of new emerging diseases; (ii) increased incidence and prevalence of preexisting infectious diseases; (iii) appearance of antimicrobialresistant strains (1). These trends occur within a context of globalization, international trade, migration, and ecological changes.

Highly pathogenic viruses have been related to some of the major pandemic and epidemics that affected the course of human history [(15); Figure 1B]. Since HIV outbreak in the 80s, we have witnessed the discovery of other highly pathogenic viruses as well as reemerging pathogens (Figure 1C), including SARS-CoV-2 responsible for the currently ongoing COVID-19 pandemic.

Vaccines have clearly been game-changers in the fight against viral diseases. Antivirals play an invaluable role mainly to control the HIV pandemics, the hepatitis $\mathrm{C}$, and several herpesviruses where vaccination has so far failed. Successful control and eradication of pandemic viruses can only be achieved by prophylactic vaccination while antivirals can decrease the morbidity and mortality related to the viral infection. However, using effective antivirals to fight pandemics or any viral disease which appears as epidemics could be pivotal to control the spread of (re)emerging viruses. The armaments (hygiene, vaccines, and antivirals) we dispose to fight against viral diseases are continuously developing to counteract not only (re)emerging viral pathogens but also endemic viral pathogens (e.g., HBV and herpesviruses) that had been infecting and co-evolved with human beings over very long time periods.

\section{VIRAL VACCINES: CORNERSTONES OF GLOBAL HUMAN HEALTH}

Though several approaches have been used to control viral disease (improved sanitation, quarantine, and vector control), successful disease prevention has occurred mostly thanks to vaccination (16). Traditional vaccination strategies, based on inactivated viral preparations or liveattenuated strains, have successfully controlled dreadful viral diseases (e.g., smallpox, polio, and measles). Smallpox eradication, thanks to massive vaccination worldwide, is one of the greatest achievements of humankind. Without vaccination, it is projected that smallpox would cause as many as 5 million deaths per year, measles 2.7 million deaths and polio 600,000 deaths or paralytic cases (17).

In addition to traditional inactivated or live attenuated vaccines, virus-vectored, subunit, viral-like particle, nucleic acid-based vaccines, and rational vaccine design provide innovative technologies to surmount existing challenges of vaccine development. These novel vaccine strategies significantly provided insights into vaccine immunology, and proved extremely useful in rapid vaccine development for emerging infectious diseases. Safer formulations based on purified 
recombinant proteins led to effective vaccines against $\mathrm{HBV}$, rotavirus, and HPV. Vaccines based on nucleic acids have gained popularity as RNA and DNA vaccines can be made fast because no culture or fermentation is required, instead synthetic processes are used. Due to the simplicity of in vitro mRNA transcription, RNA vaccines can be developed and changed extremely fast and, importantly, they are safe in theory and have shown acceptable tolerability. Though they are not cost-effective, are expensive, not very easy to produce in high quantity, and hard to deliver, the enormous global collaborative research efforts and immense investments made it possible that the RNA vaccines reached commercialization in an unprecedented time and are in the frontline combating the COVID-19 pandemic.

However, for many important known viral pathogens (HIV, herpesviruses others than VZV, HCV, and RSV), effective vaccines are not available. Fully protective vaccination against influenza virus continues to be a challenge. Newer vaccine technologies are also urgently needed to combat (re)emerging viruses.

\section{CHALLENGES FOR VACCINE DEVELOPMENT AND POTENTIAL SOLUTIONS}

\section{Challenges}

Several substantial issues must be considered for viral vaccine development, at the level of basic virology and efficacy (18). A main barrier in vaccine development is pathogen variability due to: (i) huge genetic diversity among viral types (e.g., >160 different rhinovirus serotypes); (ii) antigenic shift and drift (e.g., influenza virus requiring yearly vaccine formulations); (iii) viruses with high mutation rate (e.g., HIV and HCV); (iv) zoonotic viruses (e.g., SARS-CoV, MERS, SARS-CoV-2, influenza H5N1 and H7N9). Zoonotic viruses may show limited variability in their natural hosts; however, these viruses have the potential to infect humans (an immunological naïve population). If the pathogens establish themselves in the new host, in the process of adaptation to humans, new variants will appear, increasing the viral genetic variability. Although SARS-CoV-2 shows less genetic variability than HIV or HCV, variants with higher transmissibility are being detected, raising concerns on vaccine efficacy. A major hurdle in HIV vaccine development is linked to HIV extensive genetic variability (19). For dengue vaccines, imbalanced immunity against the four serotypes is a major drawback (20).

Molecular mimicry between pathogenic viruses and human proteins may lead to immune cross-reactivity playing a role

\footnotetext{
Abbreviations: AIDS, acquired immunodeficiency syndrome; CMV, cytomegalovirus; COVID-19, coronavirus disease 2019; CRISPR/Cas9, Clustered Regularly Interspaced Short Palindromic Repeats (CRISPR) and CRISPRassociated (Cas); HAART, highly active antiretroviral therapy; HBV, hepatitis $B$ virus; HCV, hepatitis $C$ virus; HIV, human immunodeficiency virus; HPV, human papillomavirus; HTLV, human T-lymphotropic virus; MERS, Middle East respiratory syndrome; MERS-CoV, Middle East respiratory syndrome coronavirus; RSV, respiratory syncytial virus; SARS, severe acute respiratory syndrome; SARS$\mathrm{CoV}$, severe acute respiratory syndrome coronavirus; SARS-CoV-2, severe acute respiratory syndrome coronavirus $2 ; \mathrm{VZV}$, varicella-zoster virus.
}

in the etiologies of different inflammatory and autoimmune diseases as the reaction of the immune system toward the pathogenic antigens may also injure the similar human proteins. In some SARS-CoV-2 infected patients, molecular mimicry-induced adverse autoimmune-related manifestations (e.g., transverse myelitis) have been reported, highlighting the need for continuous surveillance of vaccine side-effects (21).

Antibody-dependent disease enhancement (ADE) occurs when antibodies generated during an immune response can recognize and bind to a pathogen, but they cannot prevent infection; instead, these antibodies allow the virus getting into cells or triggering harmful immunopathology. This complication has been an impediment in development of vaccines for dengue, RSV, and coronavirus disease in cats, and is a potential barrier to COVID-19 vaccines (22). Anti-SARS-CoV2 antibodies could exacerbate COVID-19 through ADE, which is a general concern for vaccine development because the mechanisms that trigger antibody protection against any virus have theoretically the potential to intensify the infection or exacerbate immune responses.

Understanding host genetic variability is another important challenge in vaccinology as host gene polymorphisms influence vaccine-specific immune responses, a phenomenon demonstrated for HBV, measles, rubella, mumps, smallpox, cytomegalovirus, and influenza virus (18).

Ethnicity, race, age, and sex can impact vaccine-induced immune responses, leading to variability of immune responses. The lower immune response to vaccination in the elderly, young children, and patients with congenital or acquired immunodeficiency needs special consideration.

Immunodominance is a crucial principle in host response to viral infections since the immune system recognizes and respond only to a small set of immunodominant epitopes despite the generation of hundreds of distinct antigenic peptides. Pathogens exploits host immunodominance by modifying immunodominant epitopes allowing the virus the evasion of host immune responses $(23,24)$.

Due to the high complexity of the human immune system, we have an incomplete understanding of immunity development at the system-level. For some pathogens, e.g., CMV, we still do not fully comprehend how protective immunity is conferred and therefore, we are unable to reliable predict the outcome of a natural infection or of vaccination.

Neutralizing antibodies serve as a correlate of immunity for many viruses (e.g., rubella) but for some pathogens, antibody presence is clearly not a correlate of immunity/protection since infected individuals are not protected against disease despite development of antibodies. Lack of correlates of immunity/protection for some pathogens, e.g., HIV, CMV, is an important barrier in vaccine development.

Another big challenge is vaccination acceptance because of fear of some individuals that vaccines may cause diseases (e.g., autism or multiple sclerosis), despite lack of scientific evidence. A growing vaccine hesitancy movement in USA and Europe in the last years has led to decreased vaccination rates causing an increase in global measles cases (>400,000 in 2019), reversing the progress done over the last decades in measles eradication (25). 


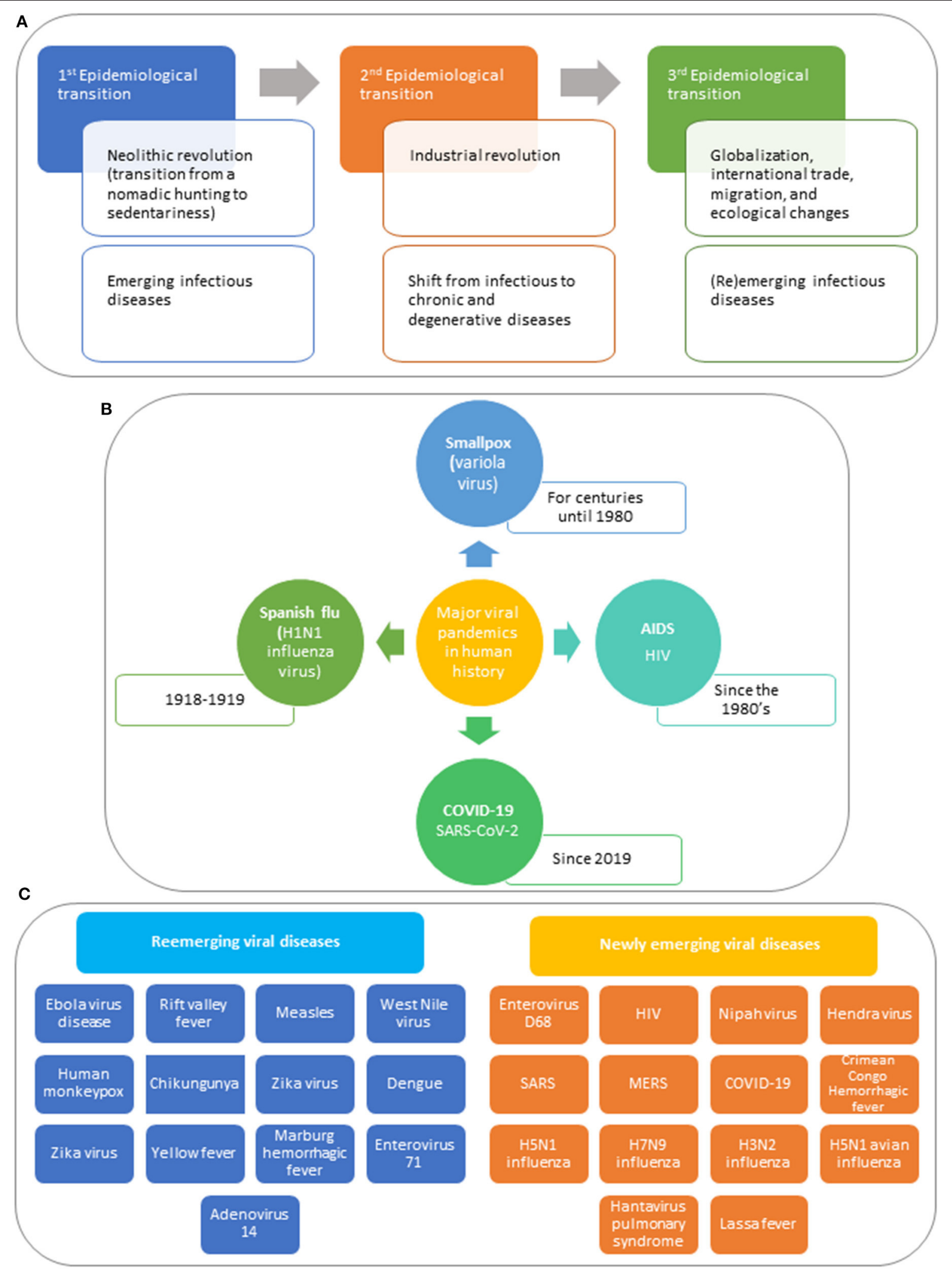

FIGURE 1 | The perpetual challenge of viral infections. (A) Epidemiological transitions. Communicable diseases became known when human beings organized in prehistoric hunter-gatherer societies. About 10,000 years ago, the Neolithic Revolution, also called the first agricultural revolution occurred, which was characterized by a transition from a nomadic hunting and gathering lifestyle to sedentariness (1). The introduction of agriculture and of domestication and herding of animals created (Continued) 
FIGURE 1 | the conditions for a rise in infectious diseases, known as the first epidemiological transition. Infectious diseases such as malaria, tuberculosis, leprosy, influenza, and smallpox were already present several millennia ago. In the mid-nineteenth century, the second epidemiologic transition took place with the industrial revolution, featured by a shift from infectious to chronic and degenerative diseases. In the last decades, emerging and reemerging infectious diseases marked the beginning of the third epidemiological transition, characterized by three main trends: (i) identification of a substantial number of new diseases associated with significant morbidity and mortality in human beings; (ii) an increase in incidence and prevalence of preexisting infectious diseases thought to be under control; (iii) appearance of antimicrobial-resistant strains that are difficult to manage (1). These trends occur within a context of globalization, international trade, migration, and ecological changes that affect the climate and the environmental equilibrium. (B) Major viral pandemics in human history. Smallpox, one of the greatest scourges in human history, first appeared in agricultural communities in northeastern Africa about 10,000 B.C.E, spreading throughout the Old World (2). Smallpox was endemic to Europe, Asia, and Africa for centuries, killing millions of people. Variola virus, caused devastation on native populations because the indigenous populations lacked natural immunity to smallpox, wiping out 90 to $95 \%$ of the indigenous people over a century. It is unknown how many people smallpox has killed throughout centuries, but only in the twentieth century, smallpox estimated mortality is of 300 to 500 million people. Smallpox could be eradicated thanks to massive vaccination campaigns $(3,4)$. The 1918 pandemic influenza (Spanish flu), caused by an antigenically distinct H1N1 influenza virus, occurred in three different waves, starting in March 1918, and waning by the summer of 1919 (5). The virus infected about one-third of the planet's population ( 500 million people at that time) and made an estimated 50 million victims. During the 1918 influenza pandemic, the cause of the Spanish flu was unknown; there were no antivirals to treat the disease or vaccines to prevent infection, no antibiotics for therapy of secondary bacterial infections, and no healthcare notion. The main response to the Spanish flu was based on physical and social distancing, isolation, and quarantine. The Spanish Flu marked an inflection point in global health as the concept of socialized medicine emerged and the importance of coordinating public health at the international level was recognized. Since the discovery of HIV/AIDS [beginning of 1980's, more than 77 million people have been infected with this virus, and about 35 million people have died of AIDS $(6,7)$. To date, around 37 million worldwide live with HIV, of whom 22 million are on treatment. Although there is no cure for AIDS and no vaccines to prevent HIV, HAART can successfully control HIV. The HIV/AIDS pandemic have had a negative influence on the global economy, though Africa, where the highest percentage of HIV/AIDS cases occur, was particularly affected. Developed countries engaged with the developing world to give access to antiretroviral therapy and to establish a health care infrastructure. In December 2019, a cluster of pneumonia cases of unknown etiology was reported in Wuhan City (Hubei province, China) (8, 9). By mid-January 2020, a novel coronavirus, named SARS-CoV-2, was identified as the etiologic agent of this outbreak. The COVID-19 pandemic is not only a health crisis; it affects our society and the economy. The impact of the pandemic will differ from country to country, but at a global scale, it will increase poverty and inequalities, propelling the amount of people living in conditions of extreme poverty in the next decade. COVID-19 is changing the trajectory of development the world had before the emergence of SARS-CoV-2. (C) Examples of newly emerging and reemerging viral diseases in the past decades. The (re)emergence of infectious diseases is mostly the consequence of the changes humans introduced in nature. Most zoonosis have occurred because of an increase contact between human beings with animals, linked to the agricultural revolution of the last decades (10). Bush meat handling and consumption of non-human primates is an effective route for the transmission of zoonotic pathogens into human populations. Several viruses, such as HIV/AIDS (via SIV) and HTLV, associated with leukemia, lymphoma, and myelopathy, have been related to pathogens of non-human primates. Bush meat is also linked to the reemergence of Ebola virus in 2015-2016. Since the 1918-1919 Spanish flu, other less severe influenza pandemics have occurred in 1957 (H2N2 Asian Flu), 1968 (N3H2 Hong Kong Flu), and 2009-2010 (H1N1 Swine Flu) (11). Besides, several epidemics caused by novel avian influenza viruses, including the Asian H7N9 virus, and the Asian H5N1 virus, which normally do not infect humans, have occurred after exposure to infected poultry or contaminated environments. Vector-borne diseases are highly sensitive to climate changes (10). Arthropod-borne viruses, including dengue virus, chikungunya virus Zika virus, West Nile virus, and Crimean Congo hemorrhagic fever virus have reemerged in many tropical and subtropical areas in the two last decades. In addition to SARS-CoV-2, two other coronaviruses crossed animal-human barriers and emerged as major human pathogens in the twenty-first century, causing deadly diseases in humans (12). SARS-CoV was reported by China in 2002 and MERS-CoV by Saudi Arabia in 2012. Unfortunately, no actions were taken to reduce the risks of emergence of new coronaviruses following these two alerts. The outbreaks of novel human coronaviruses are the result of the interactions between humans and animals. Preparedness to face the appearance of other pandemics caused by another coronavirus, an influenza virus, a paramyxovirus, a flavivirus, or any other known or a new zoonotic virus is mandatory. Lessons can be learned from previous coronavirus outbreaks and from the deadly Spanish Flu pandemic, taking into account the (dis)similar biological features and clinical characteristics of the illnesses these viruses caused $(13,14)$. The COVID-19 pandemic was unpredictable and overwhelming, completely, and radically changing our lives and lifestyle, causing traumatic events comparable to a war world for some people. It has resulted in millions of people infected around the World and thousands of deaths. What is more stressful is that there is still a great uncertainty on how this crisis will evolve and on the possible post-crisis scenarios. The efficacy and public uptake of the already available vaccines and of those arriving to the market soon as well as the development of a specific antiviral treatment will be key factors for the recovery of this unprecedented global health crisis.

\section{Efforts to Overcome the Challenges}

Viral genomics is of great utility for determination of viral strains diversity, virulence factors identification, selection of conserved regions, construction of vectors, generation of recombinant proteins, attenuation of vaccine strains, and design of nucleicacid based vaccines. Identification of genetically conserved regions allows targeting epitopes present across multiple strains and thus cross-protective immune responses, highly valuable to develop universal influenza vaccines, and pan coronavirus vaccines to be prepared for a next pandemic.

Systems biology and vaccinomics are used to understand the complex interactions of the different immune components in response to a natural infection or following vaccination. Transcriptomics, epigenomics, proteomics, and metabolomics have proven extremely valuable in understanding immune function, host-pathogen interactions, and pathogen genetics, providing insights into how vaccination can lead to antibody-dependent enhancement, a complication seen in development of vaccines for dengue, RSV and coronavirus disease in cats, and thus a potential barrier to COVID-19 vaccines (22).

A viral genetic approach together with host genetics analysis are needed to reveal the factors determining host immunodominant responses against dominant epitopes and how viruses exploits this phenomenon to evade the host immune system.

Structural vaccinology made possible mapping viral epitopes onto the three-dimensional structure of viral proteins, determination of antibody-antigen complexes (important to understand critical antibody functionality, such as neutralization) and identification of critical conserved regions (that can be targeted for more efficient immune responses).

Human genome-wide association studies are being used to delineate inter-individual variability, non-responders 
populations and impact of sex, age, race, and ethnicity in vaccine-induced immunity (26). A fundamental focus of vaccine development for immunosuppressed persons is the activation of innate immune response without using lifeattenuated vaccines. To increase the immunogenicity of subunit vaccines, the use of adjuvants is necessary. Since the first generation of adjuvants (aluminum salts and mineral oil-in-water emulsions), much efforts have been done toward delivery of novel safe adjuvants (27).

Although several conventional viral vaccines (attenuated or inactivated pathogens or protein subunits) have been produced, new vaccines are being developed, consisting of nucleic acid that encode the desired antigen(s). These novel vaccines are designed based on data obtained from systems biology, adjuvant discovery, new formulations and vaccine vectors, immunization routes, host factors studies, and results from animal models. CRISPR/Cas9 genome editing technology was used to excise virulence genes and create viral vaccines (18).

Monitoring of vaccine safety is a necessity and genetic approaches (immunogenomics, system biology, computational modeling and bioinformatics, termed "adversomics") are being used to better understand genetic and non-genetic determinants of aberrant vaccines responses at molecular level (18). Significant efforts need to be conducted at the scientific level and public advocacy level to restore confidence in vaccines. The COVID19 pandemic has increased fear and uncertainty because of rapid SARS-CoV-2 vaccine development.

\section{ANTIVIRALS: SUCCESSFUL STORIES AND FUTURE STEPS}

The foundations of antiviral therapy began in the early 1950s with the search of new molecules capable of hindering DNA synthesis for anticancer treatments. In the 1960s, scientists had doubts that specific drugs could be found to fight viruses. Acyclovir approval (in 1982) was a breakthrough, providing proof-ofconcept that highly effective and non-toxic antivirals could be developed, pioneering a more rational approach to antiviral drug development (28).

At present, 106 drugs have been licensed for therapy of viral diseases. Despite the vast diversity of human viruses (over 200 currently known), antivirals are approved only for a handful of viruses (HIV, HCV, influenza virus, RSV, HBV, HPV, herpesviruses, and SARS-CoV-2 (29). Half of the approved antiviral drugs are used for HIV therapy. The development of effective HIV treatments was remarkable in speed and success, boosting antiviral research. After the approval of the first antiretroviral drug in 1987, a breakthrough in HIV therapy was the introduction in mid-1990's of HAART able to reduce AIDSrelated deaths by $60-80 \%$ (30). Although tremendous efforts have been done in antiretroviral therapy, culminating in the one-pill once-a-day regimens, there are still about one million people dying of AIDS per year.

The antiviral drugs available to treat viral infections have saved tens of millions of human lives over the last decades, and they will continue to be a cornerstone for treatment of current as well as (re)emerging viral infections. Although great achievements have been made, there are big challenges to undertake. Antiviral compounds displaying broad-spectrum activity against different virus subtypes or genotypes should be developed. Several antiHCV drugs show activity against genotype 1 but not against other genotypes. However, acyclovir, foscarnet, ribavirin, tenofovir, and cidofovir are used for therapy of infections caused by different viruses, indicating that development of broad-spectrum antivirals is doable, which is of extreme importance as first-line measure for (re)emergent new viruses.

We also still need antiviral drugs that are more potent than the currently available antivirals to suppress completely viral replication, limiting drug-resistance, a major cause of HIV therapy failure. At present, we are unable to eradicate herpesviruses and HIV from their latent state, hence we need to tackle the viral genomes that persist in the host. Effective treatments to treat co-infections with different viruses (HBV/HIV, HCV/HIV) need to be developed. Importantly, we need cost-effective and non-toxic antivirals.

\section{STRATEGIES AND TRENDS IN ANTIVIRAL RESEARCH}

Although viruses are intracellular parasites that rely on the host cells for replication sharing many stages of their replicative cycle with cellular life cycles, most antivirals target specific steps of virus multiplication. It is challenging to identify compounds that specifically shut down viral replication without harming the host cells. In addition, some viruses may rapidly acquire resistance to a drug $(31,32)$.

High-throughput screening is based on evaluation of hundreds of thousands of natural and synthetic chemicals for antiviral activity and toxicity with the aim at identifying candidate compounds (33). Thanks to the advances in molecular virology enabling production of functional viral proteins for biochemical testing, antivirals for viruses that cannot be grown in cell culture can be discovered. Another alternative for the discovery of new antivirals is rational drug design where modifications are made to a chemical structure with the aim of increasing its antiviral potency and/or reducing its cytotoxicity.

Modeling programs are used to computationally screen chemicals for predicting interactions with viruses, reducing lab work. There are currently being reported in silico docking studies or other computer-based predictions of antiviral activity without support of biological assays (34). Although this is an interesting approach for accelerating and reducing drug discovery and development process costs, theoretical findings should be sustained by biological data to provide promising new insights and speed up the drug discovery workflow. Computational drug discovery methods (e.g., molecular docking, molecular similarity calculation, pharmacophore modeling and mapping, sequencebased virtual screening, and de novo design) can be applied at different stages in drug discovery and development, being particularly important for target identification in conjunction with biological validation. 


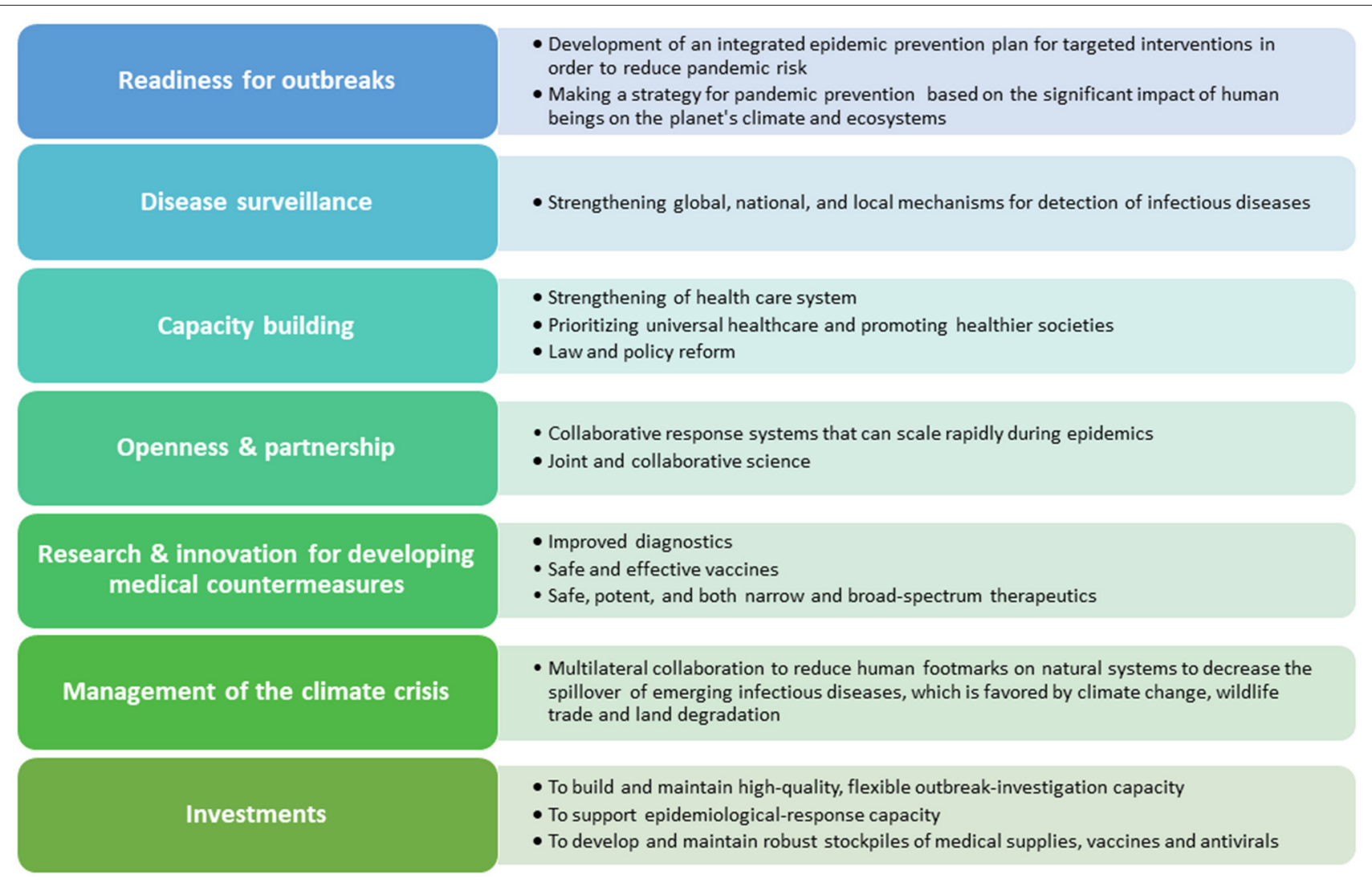

FIGURE 2 | Pillars to improve pandemic preparedness and management.

Preclinical evaluation in an animal model before clinical trials is a critical step (33). Unfortunately, many initially promising drugs fail to show efficacy in vivo, or prove toxic to animals, and an important number of candidates fail in clinical trials. The use of in vitro systems that are not representative of the in vivo situation can explain, at least in part, this failure. Many antiviral tests use a cell type that is different from the one the virus infects in the host. Numerous assays utilize established cell lines, which are immortalized cells or even worse transformed cells with alterations in several cellular pathways. Drug metabolism and viral replication can be different in these cells than in normal primary cells. Cell culture adapted viral strains that have acquire genetic changes or loose part of their genome are often used. Most in vitro systems are based on cells grown in monolayers that do not always behave as in the natural host. Human-specific viruses, for which no animal model exist, pose a major problem for drug-preclinical testing and data obtained in animals cannot directly be extrapolated to the human situation. Therefore, we need to develop and validate novel in vitro tests that can mimic the in vivo situation, such as three-dimensional cultures, and to develop animal models for which data can be extrapolated more faithfully to the human situation.

The process required to develop a new antiviral drug is complex, highly expensive (an estimated 2.8 billons for a single drug) and long (on average 12 years). In emergency situations
(COVID-19 pandemic), the search for an effective treatment can be accelerated through drug repurposing (35); a strategy that takes a drug already licensed for therapy of another disease and repurpose it to be used to treat an infection caused by a novel virus. Because safety and pharmacokinetic profile of the potential drug-candidates are already known, a limited number of in vitro studies to evaluate their antiviral activity and specificity is mandatory.

Most approved antiviral drugs are small molecules that inhibit the replication of the viral genomes. During the last years, efforts are being made to target different steps of the viral replicative cycle (29). Besides, new treatment perspectives including nanoparticles, monoclonal antibodies, and the CRISPR/Cas9 system are being explored (36). Nanoparticles are used in different formats and composition as delivery system of antiviral molecules to improve their therapeutic efficacy and reduce their toxicity (37). Both polyclonal and monoclonal neutralizing antibodies can be potentially used as antivirals because of their capacity to target specific epitopes. Despite some limitations of neutralizing antiviral antibodies as therapeutic agents, they have potential for prevention and treatment of several viral infections (Ebola, HIV, chikungunya virus, MERS-CoV, SARS-CoV, and SARS-CoV-2) (38). The CRISPR/Cas-9 technology is being used in different antiviral strategies, including: (i) modification of viral entry receptors, (ii) knock-down of host viral factors, (iii) induction of host restrictions factors, (iv) excision and removal of 
viral genomes that are integrated in the host genome or persisting as episomes $(39,40)$.

To date, only a few approved antivirals target the host systems (28). Even though much efforts have been directed toward the development of host cell-directed therapeutics with the advantage that they can potentially have a broad spectrum antiviral activity and a high genetic barrier to resistance, results have been disappointing, mostly due to toxicity issues. However, targeting host factors remains an active research area. Proteomics and genome-wide CRISPR screens can be used to reveal host factors critical for viral Infection $(41,42)$.

\section{ANTIVIRALS AND VACCINES: CHALLENGING FUTURE}

We have experienced the global progress in infectious diseases control in the last years thanks to improved sanitation, effective vaccination and use of effective antiviral therapy. However, infectious diseases remain a leading cause of mortality worldwide, new viral diseases are emerging and known diseases are reemerging. Though new and improved vaccines and antivirals are being made, several endemic and (re)emerging viral diseases remain a threat for humankind. It is almost certain that we will face an increased number of viral diseases. Pathogenic viruses can move fast and unexpectedly spill over into new populations. The major epidemics and pandemics seen in the last decades and the social and environmental changes promoted by humans contributing to the emergence of these viruses forecast the appearance of a next pandemic. At present, politicians and key decision-makers pay full attention to COVID-19 but we need to learn from this cruel experience to rethink our approaches to future national and international preparedness (Figure 2).

\section{REFERENCES}

1. Zuckerman MK, Harper KN, Barrett R, Armelagos GJ. The evolution of disease: anthropological perspectives on epidemiologic transitions. Glob Health Action. (2014) 7:23303. doi: 10.3402/gha.v7.23303

2. Graversen VK, Hamichi SE, Gold A, Murray TG. History through the eyes of a pandemic. Curr Opin Ophthalmol. (2020) 31:538-48. doi: 10.1097/ICU.0000000000000711

3. Breman JG, Arita I. The confirmation and maintenance of smallpox eradication. $N$ Engl J Med. (1980) 303:126373. doi: 10.1056/NEJM198011273032204

4. Barquet N, Domingo P. Smallpox: the triumph over the most terrible of the ministers of death. Ann Intern Med. (1997) 127 (8 Pt 1):635-42.

5. Nickol ME, Kindrachuk J. A year of terror and a century of reflection: perspectives on the great influenza pandemic of 1918-1919. BMC Infect Dis. (2019) 19:117. doi: 10.1186/s12879-019-3750-8

6. Boutayeb A. The impact of HIV/AIDS on human development in African countries. BMC Public Health. (2009) 1(Suppl. 9):S3. doi: 10.1186/1471-2458-9-S1-S3

7. Beheshti A, Vanderburg C, McDonald JT, Ramkumar C, Kadungure $\mathrm{T}$, Zhang $\mathrm{H}$, et al. A circulating microRNA signature predicts age-based development of lymphoma. PLoS ONE. (2017) 12:e0170521. doi: 10.1371/journal.pone.0170521

8. Coronaviridae Study Group of the International Committee on Taxonomy of Viruses. The species Severe acute respiratory syndrome-related coronavirus:
Of most importance, preparedness to manage the next pandemics needs to encompass the implementation of the discovery of novel vaccine and therapeutic strategies that can be rapidly made available, which will require important research efforts and investments, without neglecting research on known viral pathogens. Thanks to years of research on vaccine development for related coronaviruses, faster ways to manufacture vaccines, and colossal investments allowing the run of multiple trials in parallel, and regulatory agencies moving more rapidly than usual, COVID-19 vaccines could be delivered so quickly. This success might be beneficial for the development of vaccines against other pathogens though researchers were fortunate with SARS-CoV-2 as this virus does not mutate so fast and does not have sophisticated strategies for hindering the human immune system, unlike HIV, herpesviruses, or influenza. Herpesviruses have higher and multiple immune evasion capabilities (e.g., actively block antibodies from binding) and HIV and influenza have faster mutation rates. In contrast to vaccination, antiviral drugs for SARS-CoV-2 failed to deliver on their expectations. To date, clinically approved antivirals are available for only a limited series of viruses and it is imperative that this precarious situation changes and hopefully, the COVID19 pandemic will boost antiviral research. SARS-CoV-2/COVID19 pandemic has underscored the pivotal need for antivirals that can be rapidly employed before a (re)emerging virus reaches the global emergency status and more efforts should be put into the development of antivirals.

\section{AUTHOR CONTRIBUTIONS}

The author confirms being the sole contributor of this work and has approved it for publication. classifying 2019-nCoV and naming it SARS-CoV-2. Nat Microbiol. (2020) 5:536-44. doi: 10.1038/s41564-020-0695-Z

9. Gralinski LE, Menachery VD. Return of the coronavirus: 2019-nCoV. Viruses. (2020) 12:135. doi: 10.3390/v12020135

10. Sabin NS, Calliope AS, Simpson SV, Arima H, Ito H, Nishimura $\mathrm{T}$, et al. Implications of human activities for (re)emerging infectious diseases, including COVID-19. J Physiol Anthropol. (2020) 39:29. doi: 10.1186/s40101-020-00239-5

11. Trovao NS, Nelson MI. When pigs fly: pandemic influenza enters the 21st century. PLoS Pathog. (2020) 16:e1008259. doi: 10.1371/journal.ppat.1008259

12. Cui J, Li F, Shi ZL. Origin and evolution of pathogenic coronaviruses. Nat Rev Microbiol. (2019) 17:181-92. doi: 10.1038/s41579-018-0118-9

13. Petersen E, Koopmans M, Go U, Hamer DH, Petrosillo N, Castelli F, et al. Comparing SARS-CoV-2 with SARS-CoV and influenza pandemics. Lancet Infect Dis. (2020) 20:e238-44. doi: 10.1016/S1473-3099(20)30484-9

14. Zhu Z, Lian X, Su X, Wu W, Marraro GA, Zeng Y. From SARS and MERS to COVID-19: a brief summary and comparison of severe acute respiratory infections caused by three highly pathogenic human coronaviruses. Respir Res. (2020) 21:224. doi: 10.1186/s12931-020-01479-w

15. Morens DM, Daszak P, Markel H, Taubenberger JK. Pandemic COVID-19 joins history's pandemic legion. mBio. (2020) 11:e0081220. doi: $10.1128 / \mathrm{mBio} .00812-20$

16. Hajj Hussein I, Chams N, Chams S, El Sayegh S, Badran R, Raad M, et al. Vaccines through centuries: major cornerstones of global health. Front Public Health. (2015) 3:269. doi: 10.3389/fpubh.2015.00269 
17. Hinman AR. Global progress in infectious disease control. Vaccine. (1998) 16:1116-21. doi: 10.1016/s0264-410x(98)80107-2

18. Kennedy RB, Ovsyannikova IG, Palese P, Poland GA. Current challenges in vaccinology. Front Immunol. (2020) 11:1181. doi: 10.3389/fimmu.2020.01181

19. Ng'uni T, Chasara C, Ndhlovu ZM. Major scientific hurdles in HIV vaccine development: historical perspective and future directions. Front Immunol. (2020) 11:590780. doi: 10.3389/fimmu.2020.590780

20. Martinez DR, Metz SW, Baric RS. Dengue vaccines: the promise and pitfalls of antibody-mediated protection. Cell Host Microbe. (2021) 29:1322. doi: 10.1016/j.chom.2020.12.011

21. Halpert G, Shoenfeld Y. SARS-CoV-2, the autoimmune virus. Autoimmun Rev. (2020) 19:102695. doi: 10.1016/j.autrev.2020.102695

22. Jeyanathan M, Afkhami S, Smaill F, Miller MS, Lichty BD, Xing Z. Immunological considerations for COVID-19 vaccine strategies. Nat Rev Immunol. (2020) 20:615-32. doi: 10.1038/s41577-020-00434-6

23. Angeletti D, Gibbs JS, Angel M, Kosik I, Hickman HD, Frank GM, et al. Defining B cell immunodominance to viruses. Nat Immunol. (2017) 18:45663. doi: 10.1038/ni.3680

24. Altman MO, Angeletti D, Yewdell JW. Antibody immunodominance: the key to understanding influenza virus antigenic drift. Viral Immunol. (2018) 31:142-9. doi: 10.1089/vim.2017.0129

25. Phadke VK, Bednarczyk RA, Omer SB. Vaccine refusal and measles outbreaks in the US. JAMA. (2020) 324:1344-5. doi: 10.1001/jama.2020.14828

26. Bragazzi NL, Gianfredi V, Villarini M, Rosselli R, Nasr A, Hussein A, et al. Vaccines meet big data: state-of-the-art and future prospects. From the classical 3Is (isolate-inactivate-inject) vaccinology 1.0 to vaccinology 3.0, vaccinomics, and beyond: a historical overview. Front Public Health. (2018) 6:62. doi: 10.3389/fpubh.2018.00062

27. Apostolico Jde S, Lunardelli VA, Coirada FC, Boscardin SB, Rosa DS. Adjuvants: classification, modus operandi, and licensing. I Immunol Res. (2016) 2016:1459394. doi: 10.1155/2016/1459394

28. De Clercq E, Li G. Approved antiviral drugs over the past 50 years. Clin Microbiol Rev. (2016) 29:695-747. doi: 10.1128/CMR.0 0102-15

29. Tompa DR, Immanuel A, Srikanth S, Kadhirvel S. Trends and strategies to combat viral infections: a review on FDA approved antiviral drugs. Int J Biol Macromol. (2021) 172:524-41. doi: 10.1016/j.ijbiomac.2021. 01.076

30. McColl ER, Kojovic D, Piquette-Miller M. Battling the HIV/AIDS epidemic: triumphs and barriers. Clin Pharmacol Ther. (2018) 104:10426. doi: $10.1002 /$ cpt.1202

31. Littler E, Oberg B. Achievements and challenges in antiviral drug discovery. Antivir Chem Chemother. (2005) 16:15568. doi: $10.1177 / 095632020501600302$
32. Chaudhuri S, Symons JA, Deval J. Innovation and trends in the development and approval of antiviral medicines: 1987-2017 and beyond. Antiviral Res. (2018) 155:76-88. doi: 10.1016/j.antiviral.2018.05.005

33. Adamson CS, Chibale K, Goss RJM, Jaspars M, Newman DJ, Dorrington RA. (2021) Antiviral drug discovery: preparing for the next pandemic. Chem Soc Rev. doi: $10.1039 / \mathrm{d} 0 \mathrm{cs} 01118 \mathrm{e}$

34. Patel H, Kukol A. Integrating molecular modelling methods to advance influenza A virus drug discovery. Drug Discov Today. (2021) 26:50310. doi: 10.1016/j.drudis.2020.11.014

35. Garcia-Serradilla M, Risco C, Pacheco B. Drug repurposing for new, efficient, broad spectrum antivirals. Virus Res. (2019) 264:22-31. doi: 10.1016/j.virusres.2019.02.011

36. Soppe JA, Lebbink RJ. Antiviral goes viral: harnessing CRISPR/Cas9 to combat viruses in humans. Trends Microbiol. (2017) 25:83350. doi: 10.1016/j.tim.2017.04.005

37. Cagno V, Andreozzi P, D'Alicarnasso M, Jacob Silva P, Mueller M, Galloux $\mathrm{M}$, et al. Broad-spectrum non-toxic antiviral nanoparticles with a virucidal inhibition mechanism. Nat Mater. (2018) 17:195-203. doi: 10.1038/nmat5053

38. Ali MG, Zhang Z, Gao Q, Pan M, Rowan EG, Zhang J. Recent advances in therapeutic applications of neutralizing antibodies for virus infections: an overview. Immunol Res. (2020) 68:325-39. doi: 10.1007/s12026-020-09159-Z

39. Goncalves BC, Lopes Barbosa MG, Silva Olak AP, Belebecha Terezo N, Nishi L, Watanabe MA, et al. Antiviral therapies: advances and perspectives. Fundam Clin Pharmacol. (2020) 35:305-20. doi: 10.1111/fcp.12609

40. Koujah L, Shukla D, Naqvi AR. CRISPR-Cas based targeting of host and viral genes as an antiviral strategy. Semin Cell Dev Biol. (2019) 96:5364. doi: 10.1016/j.semcdb.2019.04.004

41. Wei J, Alfajaro MM, DeWeirdt PC, Hanna RE, Lu-Culligan WJ, Cai WL, et al. Genome-wide CRISPR screens reveal host factors critical for SARS-CoV-2 infection. Cell. (2021) 184:76-91.e13. doi: 10.1016/j.cell.2020.10.028

42. Muller KH, Kakkola L, Nagaraj AS, Cheltsov AV, Anastasina M, Kainov DE. Emerging cellular targets for influenza antiviral agents. Trends Pharmacol Sci. (2012) 33:89-99. doi: 10.1016/j.tips.2011.10.004

Conflict of Interest: The author declares that the research was conducted in the absence of any commercial or financial relationships that could be construed as a potential conflict of interest.

Copyright (C) 2021 Andrei. This is an open-access article distributed under the terms of the Creative Commons Attribution License (CC BY). The use, distribution or reproduction in other forums is permitted, provided the original author(s) and the copyright owner(s) are credited and that the original publication in this journal is cited, in accordance with accepted academic practice. No use, distribution or reproduction is permitted which does not comply with these terms. 